\title{
Articulatory and acoustic adaptation to palatal perturbation
}

Mélanie Thibeault, Lucie Ménard, Shari R. Baum, Gabrielle Richard, and David H. McFarland

Citation: The Journal of the Acoustical Society of America 129, 2112 (2011); doi: 10.1121/1.3557030

View online: https://doi.org/10.1121/1.3557030

View Table of Contents: https://asa.scitation.org/toc/jas/129/4

Published by the Acoustical Society of America

\section{ARTICLES YOU MAY BE INTERESTED IN}

Incomplete compensation to articulatory perturbation

The Journal of the Acoustical Society of America 97, 1865 (1995); https://doi.org/10.1121/1.412060

The development of speech adaptation to an artificial palate

The Journal of the Acoustical Society of America 102, 2353 (1997); https://doi.org/10.1121/1.419619

Speech compensation to structural modifications of the oral cavity

The Journal of the Acoustical Society of America 100, 1093 (1996); https://doi.org/10.1121/1.416286

Sensorimotor adaptation to feedback perturbations of vowel acoustics and its relation to perception

The Journal of the Acoustical Society of America 122, 2306 (2007); https://doi.org/10.1121/1.2773966

Electropalatographic, acoustic, and perceptual data on adaptation to a palatal perturbation

The Journal of the Acoustical Society of America 119, 2372 (2006); https://doi.org/10.1121/1.2173520

Compensation strategies for the perturbation of the rounded vowel [u] using a lip tube: A study of the control space in speech production

The Journal of the Acoustical Society of America 98, 2428 (1995); https://doi.org/10.1121/1.413277

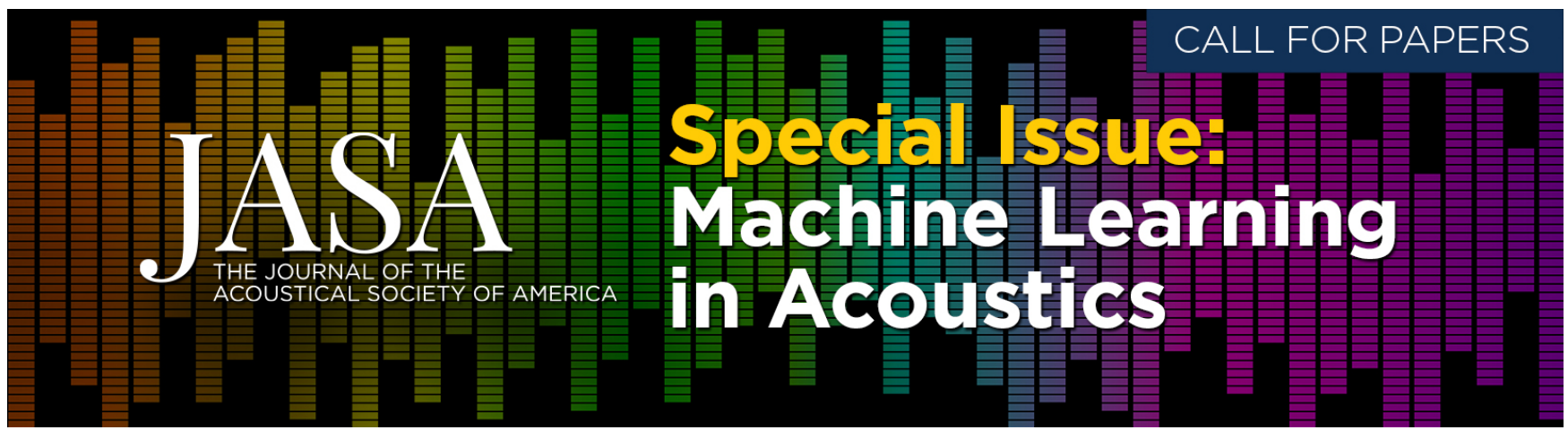




\title{
Articulatory and acoustic adaptation to palatal perturbation
}

\author{
Mélanie Thibeault ${ }^{\mathrm{a})}$ and Lucie Ménard \\ Département de linguistique, Université du Québec à Montréal, Case postale 8888, Succursale Centre-Ville, \\ Montréal, Québec, H3C 3P8, Canada \\ Shari R. Baum \\ School of Communication Sciences and Disorders, McGill University, 1266 Pine Avenue West, Montréal, \\ Québec, H3G 1A8, Canada
}

\author{
Gabrielle Richard \\ Département de réadaptation, Université Laval, 1050 avenue de la Médecine, Québec, Québec, GIV 0A6, \\ Canada \\ David H. McFarland \\ École d'orthophonie et d'audiologie, Université de Montréal, Case postale 6128, Succursale Centre-Ville, \\ Montréal, Québec, H3C 3J7, Canada
}

(Received 21 January 2010; revised 17 January 2011; accepted 19 January 2011)

\begin{abstract}
Previous work has established that speakers have difficulty making rapid compensatory adjustments in consonant production (especially in fricatives) for structural perturbations of the vocal tract induced by artificial palates with thicker-than-normal alveolar regions. The present study used electromagnetic articulography and simultaneous acoustic recordings to estimate tongue configurations during production of [s $\breve{s} \mathrm{k}$ ] in the presence of a thin and a thick palate, before and after a practice period. Ten native speakers of English participated in the study. In keeping with previous acoustic studies, fricatives were more affected by the palate than were the stops. The thick palate lowered the center of gravity and the jaw was lower and the tongue moved further backwards and downwards. Center of gravity measures revealed complete adaptation after training, and with practice, subjects' decreased interlabial distance. The fact that adaptation effects were found for [k], which are produced with an articulatory gesture not directly impeded by the palatal perturbation, suggests a more global sensorimotor recalibration that extends beyond the specific articulatory target. (C) 2011 Acoustical Society of America. [DOI: 10.1121/1.3557030]
\end{abstract}

PACS number(s): 43.70.Aj, 43.70.Bk [AL]

Pages: 2112-2120

\section{INTRODUCTION}

The human speech production system is highly flexible and speakers can compensate for a variety of structural and functional articulatory perturbations, including those due to normal anatomical development (such as tooth loss and growth) and those due to disease and damage of oral-articulatory structures. Understanding adaptive mechanisms of speech production has many important implications, including providing insights into the cognitive representation of speech movements, the potential role of sensory feedback in speech development and refinement, and potential links between production and perception in individual compensatory abilities. Perturbing sensorimotor system has been a valuable tool to explore various fundamental aspects of the control and coordination of movements including speech production (Elman, 1981; Houde and Jordan, 1998; Abbs and Gracco, 1984; Gracco and Abbs, 1985; Kawahara, 1995). Experimentally induced structural and functional perturbations of the oral environment, combined with targetspecific speech practice in adults, have elucidated key aspects of speech motor control and learning and have furth-

\footnotetext{
a) Author to whom correspondence should be addressed. Electronic mail: thibeault.melanie.2@courrier.uqam.ca
}

ered our understanding of the precision of speech articulatory gestures and of the specifics of their articulatory representation in the nervous system.

Much progress has been made in understanding speakers' ability to adapt to both auditory (e.g., Houde and Jordan, 1998, 2002) and articulatory or somatosensory (e.g., Abbs and Gracco, 1984; Gracco and Abbs, 1985; Honda et al., 2002; Tremblay et al., 2003) perturbations that yield novel vocal tract configurations. Of particular relevance to the present investigation is our own work in this area which has focused on, among other things, examining speakers' ability to compensate for structural perturbations of the vocal tract induced by the presence of an artificial palate with a thickerthan-normal alveolar region (e.g., Baum and McFarland, 1997, 2000; Aasland et al., 2006). In response to this structural modification, speakers have little difficulty in achieving acoustically and perceptually normal vowel targets. This is not surprising given that the palate does not substantially interfere with the vocal tract configurations required for vowel production. However, fricative consonant spectra are significantly affected by the perturbation, and such acoustic shifts are perceptually salient to listeners. Earlier studies had suggested that fricatives, and in particular [s], may require a period of weeks for complete compensation to occur (e.g., Hamlet and Stone, 1978). The fricative [s] is particularly 
resistant to adaptation apparently due to the articulatory precision required to form the appropriate tongue-palate groove shape for its accurate production. However, in recent studies, we were able to accelerate the adaptation by providing intensive target-specific practice (Baum and McFarland, 1997, 2000). After a 1 -h period of targeted [s] practice with the palate in place, analyses revealed a gradual improvement in [s] production, as reflected in shifts in fricative spectra (centroid frequencies) toward unperturbed values, as well as improved quality ratings by phonetically trained listeners. These data suggest that the cognitive representation of speech production movements is highly plastic and can be modified significantly with this intensive target-specific practice. Sensorimotor recalibration was also apparent in the presence of negative after-effects when the palate was removed (Baum and McFarland, 1997, 2000). These findings relate directly to the general motor control literature on neural plasticity and the effects of intensive exercise or practice on brain reorganization (Kleim and Jones, 2008; Ludlow et al., 2008). The findings also relate to the growing body of evidence of multiple points of interaction among a variety of sensorimotor behaviors such as speech and swallowing and even limb and speech movements (McFarland and Tremblay, 2006). These behaviors share a variety of control processes and there appear to be some aspects of global movement parameterization that extend across these seemingly divergent movement systems (Chang et al., 2009; McFarland and Tremblay, 2006). It might be argued, therefore, that the speech articulatory system would take advantage of global parameters in the face of perturbation, and that practice effects may extend beyond the specific articulatory target. This has obvious fundamental and clinical implications and deserves further experimental attention. To more fully explore potential individual differences in compensatory abilities and the focused or distributed effects of practice in modifying speech motor control processes across sound classes or gestures requires experiments that go beyond acoustic analyses and more directly assess speech motor control by recording articulatory movements.

We recently combined both acoustic and electropalatographic (EPG) measures to tap into the articulatory gestures used in the adaptive process. We observed considerable individual variability in speech adaptation in the EPG data, with some subjects showing target overshoot reflected in an increase in tongue-palate contact and/or an unexpected place of articulation, and other subjects producing target undershoot in perturbed conditions (Aasland et al., 2006).

Although much can be learned from measures of tongue contact, EPG does not provide a direct measure of tongue shape or movement. The tongue is, of course, the primary articulator in fricative production and we have yet to explore its shape and how that may change over time to yield adaptive speech production. As contrasted to acoustic measures alone, measuring tongue shape and movement allows us to move closer to the speech movements and consequently, may provide more direct assessments of the plasticity of speech production gestures as well as the potential distributed parameterization of speech motor control processes across apparently divergent speech gestures. Important insights, therefore, may be gained about compensatory abilities and other control processes involved in speech production.

To that end, the present study made use of electromagnetic articulography (EMA) and simultaneous acoustic recordings to estimate tongue configuration during production of the fricatives [s] and [ $\check{\mathrm{s}}]$ and the stops $[\mathrm{t}]$ and $[\mathrm{k}]$ in the presence of a palatal perturbation similar to that used in our previous investigations. Compensatory responses were examined after a 15-min practice period focused on alveolar consonant production in an effort to identify the articulatory means by which speakers adapt to the perturbation. As noted, our analyses also examined articulatory gestures for sounds not targeted in the practice period (i.e., [š] and [k]) to determine whether the development of novel articulatory programs results in a more global re-parameterization that extends beyond the target gestures. We hypothesize that the immediate effect of the palate will induce tongue retraction and lowering and that the primary compensatory gesture will involve tongue raising and advancement or adjustments to jaw height to appropriately direct the airstream for clear [s] production. We further hypothesize that the adaptive adjustments will affect a broad range of sounds that involve similar places of articulation; should adaptation carry over to sounds not affected by the perturbation, it would suggest a systemic reorganization to maintain distinctions across the phonetic inventory.

\section{METHODS}

\section{A. Participants}

Ten native speakers of English (six women, four men) between 18 and 35 years of age were participated in the study, which was approved by the Institutional Review Board of the Faculty of Medicine, McGill University. The subjects reported no history of speech, language, or hearing impairment.

\section{B. Stimuli and procedure}

Based on previous studies (McFarland and Baum, 1995; Baum and McFarland, 1997; Aasland et al., 2006), two artificial palates (thin and thick) were constructed for each subject from dental acrylic. The thin palate was $1 \mathrm{~mm}$ thick. The thick palate was fabricated such that a 6-mm ridge extruded near the alveolar ridge (see Fig. 1), in order to perturb normal [s] production. The palates were fitted with ball clasps to hold them in place. The predicted effect of such a perturbation is to substantially lower the centroid frequencies for [s] (McFarland and Baum, 1995; Baum and McFarland, 1997; Aasland et al., 2006).

Subjects were asked to produce ten repetitions each of [asa], [aša], [ata], and [aka] in seven different conditions. Because some studies have shown that pellets or transducers placed on the tongue (as in EMA) themselves interfere to some extent with articulation (e.g., Katz et al., 2006; Weismer and Bunton, 1999), we include a comparison of sensors-off and sensors-on to assess the potential perturbing effects of the sensors themselves. Thus, subjects were first 


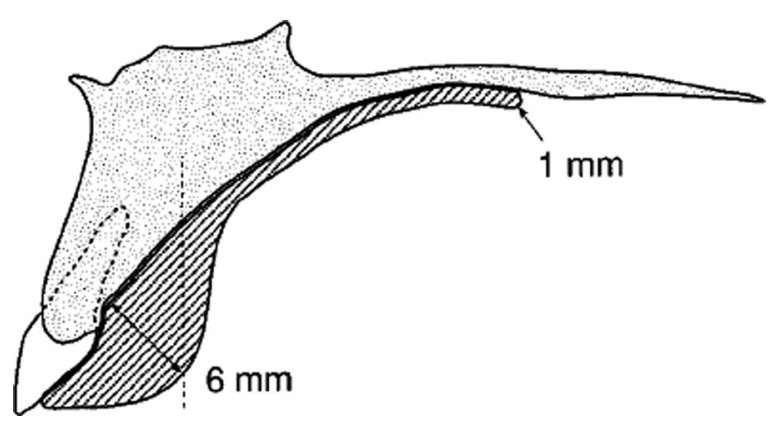

FIG. 1. Artificial thick palate (mid-sagittal view) (image taken from Baum and MacFarland, 1997).

recorded in a normal speech condition [i.e., without anything in their mouth (0OFFOFF: acoustics only)]. Subsequently, EMA sensors were secured in place and an additional recording was made (1OFFPRE). Another set of stimuli was recorded with a thin palate in place (2THINPRE) and then with a thick palate in place (3THICKPRE). The thin palate conditions were included to ensure that any adaptation found in the thick palate conditions was not simply due to the presence of an artificial palate (of any thickness), but to the specific perturbation at the alveolar ridge in the thick palate condition. Whereas practice with the thin palate could conceivably affect productions in the thick palate condition, this approach was the most logical to achieve the multiple goals of the study and was in keeping with previous investigations (e.g., Aasland et al., 2006). After these initial recordings and for a 15-min interval, participants read a series of sentences (available upon request) heavily loaded with alveolar consonants ([s z t d]) but with no back consonants [ $\check{\mathrm{s}}$ ž $\mathrm{k} g \mathrm{~g} \mathrm{w}$ ] with the thick palate in place. They were then recorded again with the thick palate (4THICKPOST), the thin palate (5THINPOST), and without any palate (6OFFPOST).

Tongue recordings were made using an EMA AG500 system (LINUX version), placed at least at $1.5 \mathrm{~m}$ from any wall, at a sampling rate of $200 \mathrm{~Hz}$ (Carstens, 2006) in a soundproof room at the Laboratoire de phonétique of the Université du Québec à Montréal. During the recordings, the subjects were seated with their heads within the EMA recording unit and with a microphone in front of them. The stimuli were presented visually in an orthographic form in a fixed random order on a computer screen located at eye level at $1.5 \mathrm{~m}$ from the subjects (to avoid interference with the EMA's electromagnetic field). The acoustic signal was recorded simultaneously with a Sony ECM-T6 microphone, placed at $30 \mathrm{~cm}$ from subject's mouth, and digitized at $44100 \mathrm{~Hz}$ using a Delta 1010 LT sound card.

Calibration of the EMA system (see Carstens, 2006) was performed before each recording with all equipment to be used during the recording was powered on. RMS values, which represent the difference in signal amplitude between predicted and recorded values (and should be smaller than 14), were all smaller than 7.53, with a mean value of 1.97 . These low RMS calibration values confirm that the relatively close proximity of the presentation screen did not affect the electromagnetic field.

Eight sensors were attached to the upper and lower lips (at the vermillion line), lower incisor (at the gum line) and

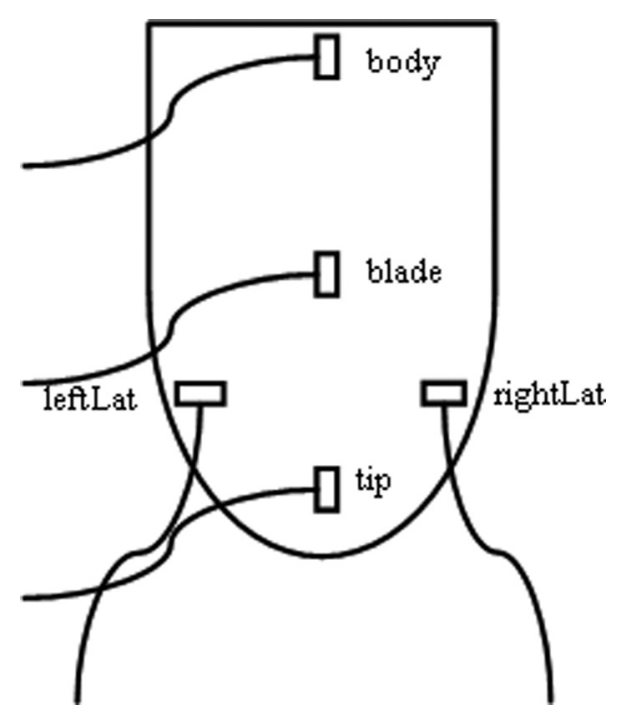

FIG. 2. Tongue sensor placement (body $=$ tongue body; blade $=$ tongue blade; tip = tongue tip; leftLat $=$ tongue lateral left; rightLat $=$ tongue lateral right).

on the tongue midline (tongue body, tongue blade, and tongue tip), and on the left and right margins between tongue blade and tongue tip (see Fig. 2). The tongue tip sensor was placed $1 \mathrm{~cm}$ back from actual tongue tip in an attempt to minimize speech perturbation. The tongue body sensor was as far back as possible and the tongue blade sensor was placed at mid-distance from the two other sensors. The margin sensors were oriented so that their theta angle (elevation) provided information about the shape of the tongue in the coronal plane. Four additional sensors were attached to the left and right mastoids and on the left and right lateral upper incisors at the gum line and used for head correction.

After the recording, position (x: back/front, y: left/right, and $\mathrm{z}$ : high/low) and orientation (phi: azimuth and theta: elevation) of each sensor through time were extracted using the LINUX version of the EMA software (Carsten's CalcPos). Sensor positions and orientation were corrected for head movements using a MATLAB procedure developed by Mark Tiede (Haskins Laboratory) based on the upper incisor sensors and the mastoid sensors.

\section{Data analysis}

At the articulatory level, sensor position and orientation were extracted at the steady state of the consonant, as determined by identifying the point of zero velocity (in the first derivative) of the tongue blade vertical movement, ${ }^{1}$ a point which usually falls near the midpoint of frication in fricatives and a few millisecond before the burst in stops. Ideally, we would have used the tongue body sensor for the velar consonant, but this was not feasible as it became unglued before the end of the recording for four subjects out of ten. Figure 3 shows an analysis window for a given sweep with the temporal landmark at which the articulatory events were extracted.

Articulatory measures included $\mathrm{x}$ (front-back) and $\mathrm{z}$ (low-high) positions of the tongue body, tongue blade, and tongue tip; $\mathrm{z}$ position of the jaw, theta angle (elevation) of 


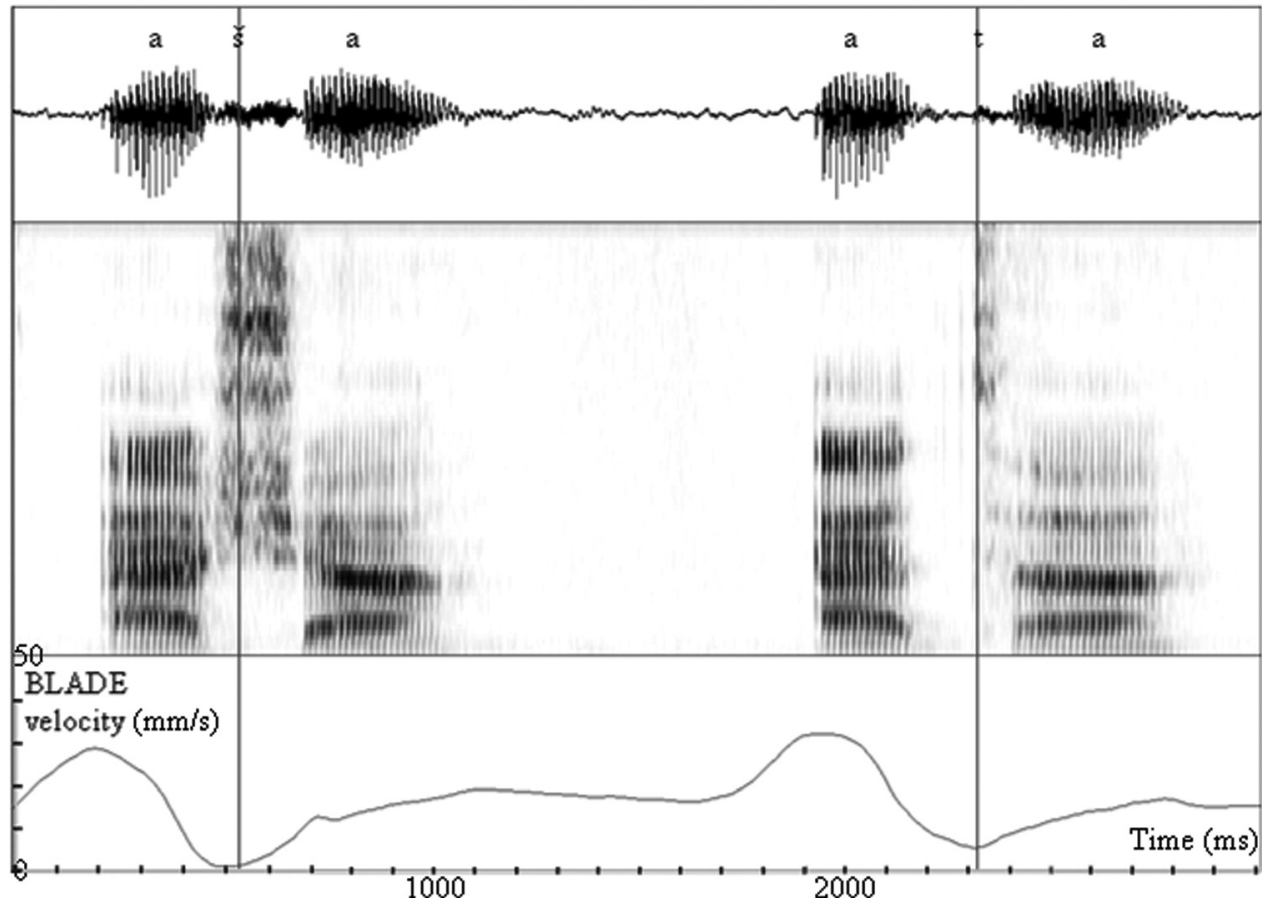

FIG. 3. Screen illustrating the marking of tongue blade minimum velocity for a fricative and a stop. the tongue left lateral sensor, interlabial distance ( $\mathrm{z}$ position of upper lip minus z position of lower lip), and lip protrusion (x position of upper lip). These measures were chosen due to their critical role in production of the sounds of interest. Because of the palatal manipulation at the alveolar ridge, it was essential to examine tongue tip position. Similarly, jaw height and interlabial distance would be affected by the perturbation, and lip protrusion might serve as a compensatory function. As tongue groove is important for [s] production, we relied on the theta angle of the left lateral sensor as a reflection of the groove. Finally, because we were interested in consonants other than [s] as well, and because the adaptive modifications might be global in nature, we also studied tongue body and tongue blade position.

At the acoustic level, two central moments were extracted from the acoustic signal: The center of gravity is the first spectral moment and is defined as a weighted average of frequency values; standard deviation (SD) is the square root of the second central moment of the spectra. Central moments were extracted using PRAAT with a $20-\mathrm{ms}$ hamming window, beginning at the same time point as the articulatory measurements for stops and centered at this point for fricatives, after the signal was low-pass filtered at half the sampling frequency $(22050 \mathrm{~Hz}$ ) with a pre-emphasis from $50 \mathrm{~Hz}$. In order to compare data across speakers, measures of center of gravity were transformed into mel units according to the following formula: $F_{\text {mel }}=550 \ln \left(1+F_{\mathrm{Hz}} / 550\right)$.

Acoustic and articulatory measures were compared across conditions to examine the direction and extent of perturbation and adaptation. For each speaker, values from the normal condition (1OFFPRE) were subtracted from the values of the perturbed conditions (2THINPRE, 3THICKPRE, 4THICKPOST, 5THINPOST) to normalize the data (2NTHINPRE, 3NTHICKPRE, 4NTHICKPOST, 5NTHIN-
POST) and to allow for comparisons of these relative change of values across speakers. ${ }^{2}$

Repeated-measures ANOVAs were conducted on the normalized values for each consonant separately, with two palate conditions (thin and thick) and two practice conditions (pre- and post-practice intervals). Tukey post hoc tests were conducted to assess any interactions revealed by the ANOVAs. In addition, paired $t$-tests were conducted comparing the OOFFOFF and 1OFFPRE acoustic measures to assess the perturbing effect of the EMA sensors, and comparing the 1OFFPRE and 6OFFPOST measures to assess the presence of an after-effect. In this paper, we consider as an after-effect a significant difference between the above mentioned conditions only for those parameters showing an adaptation.

\section{RESULTS}

\section{A. Acoustic results}

To first determine whether the presence of the EMA sensors alone may adversely affect articulation, we compared the recordings made prior to affixing the sensors (0OFFOFF) to those made immediately after affixing them (1OFFPRE). As can be seen in Fig. 4, acoustic measures were highly similar across the two conditions. In fact, paired $t$-tests revealed that the sensors had a significant influence only on $[\mathrm{s}]$ center of gravity $[F(1,7)=5.849, p \leq 0.05$; see Fig. 4]. The presence of the sensors resulted in a reduction of the center of gravity by $121 \mathrm{mel}$. As will be seen later, the palates induced a substantially larger modification of [s] center of gravity that went beyond these small changes due to the presence of the EMA sensors. We are confident that our perturbation findings are not simply because of the presence of the EMA sensors alone. 

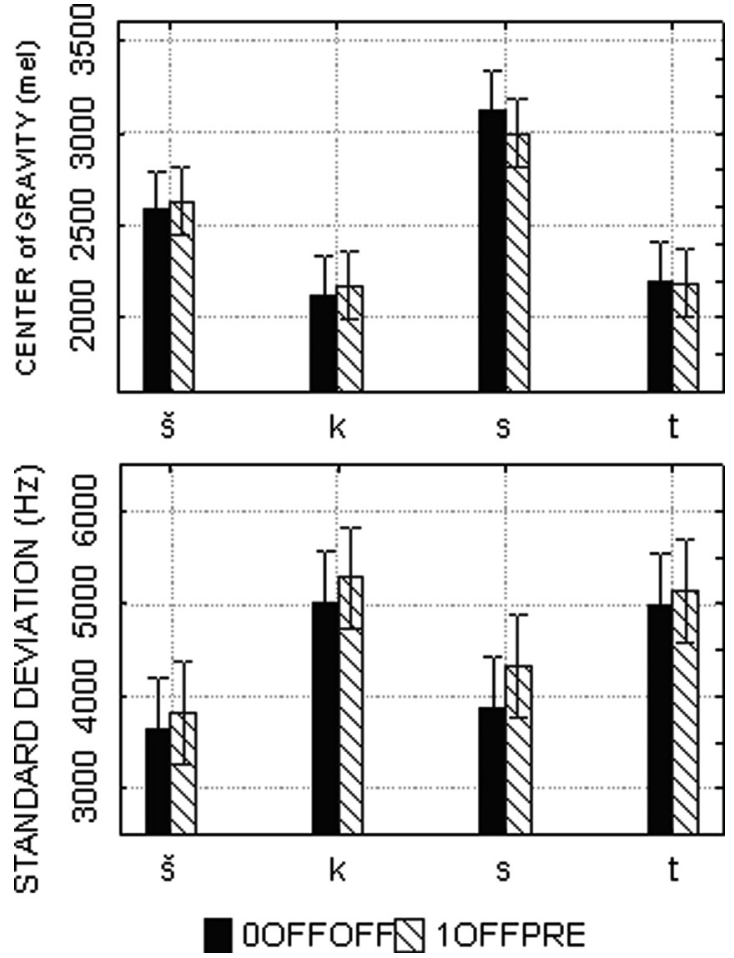

FIG. 4. Average values and SD of center of gravity and SD for the four consonants. Data are shown for the no sensor condition (OOFFOFF) and the no palate condition before practice (1OFFPRE).

Presented in Fig. 5 are the results of acoustic measures, with the normal (1OFFPRE) condition providing the zero reference point; consequently, a positive value corresponds to an increase relative to the normal condition, and a nega-

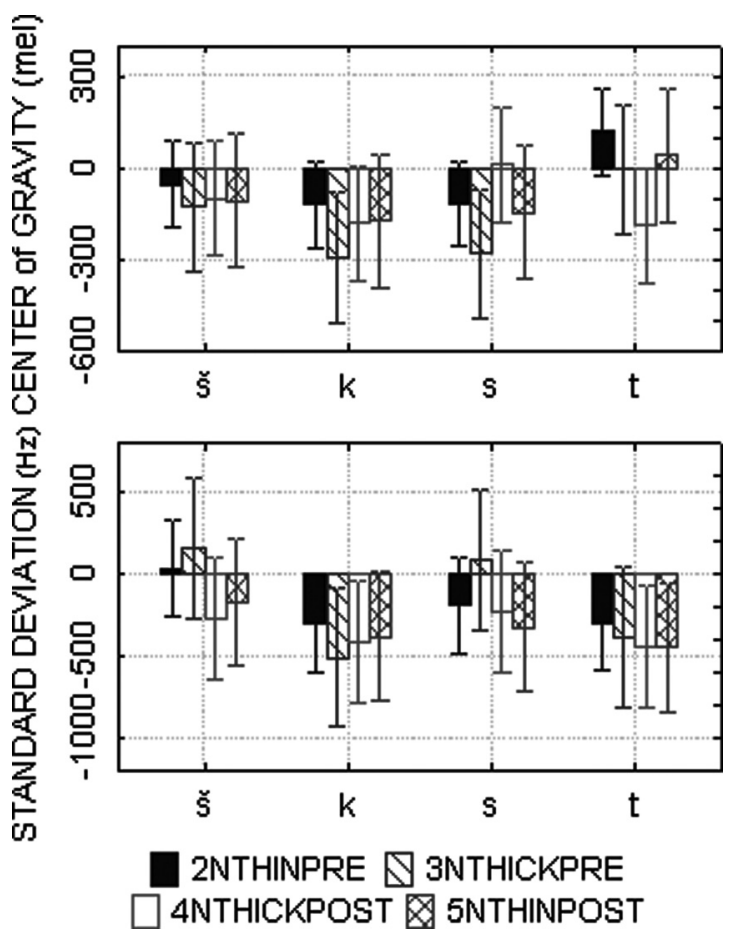

FIG. 5. Average values and SD of within-speaker normalized values of center of gravity and SD for the four consonants. Data are shown for the perturbed condition (thin palate preperturbation-2NTHINPRE, thick palate preperturbation 3NTHICKPRE, thick palate post-perturbation 4NTHICKPOST, thin palate post-perturbation 5NTHINPOST).
TABLE I. Results of repeated-measure ANOVAs and paired $t$-test conducted on the two acoustic parameters: Center of gravity (CofG) and standard deviation $(\mathrm{SD})(* p \leq 0.05, * * p \leq 0.01)$.

\begin{tabular}{|c|c|c|c|c|c|c|c|c|c|c|c|c|c|c|c|c|}
\hline \multirow[b]{3}{*}{ Dep. var. } & \multicolumn{12}{|c|}{ ANOVA } & \multicolumn{4}{|c|}{ Paired $t$-test } \\
\hline & \multicolumn{4}{|c|}{ Practice } & \multicolumn{4}{|c|}{ Palate } & \multicolumn{4}{|c|}{$\begin{array}{c}\text { Interaction } \\
\text { practice } \times \text { palate }\end{array}$} & \multicolumn{4}{|c|}{ After-effect } \\
\hline & $\check{S}$ & $\mathrm{k}$ & $\mathrm{s}$ & $\mathrm{t}$ & $\check{s}$ & $\mathrm{k}$ & $\mathrm{s}$ & $\mathrm{t}$ & $\check{\mathrm{S}}$ & $\mathrm{k}$ & $\mathrm{s}$ & $\mathrm{t}$ & $\check{s}$ & $\mathrm{k}$ & $\mathrm{s}$ & $\mathrm{t}$ \\
\hline CofG & & & $*$ & $*$ & & & & & & & & & * & $*$ & $*$ & \\
\hline SD & ** & & $*$ & & & & & & & & & & & & & $*$ \\
\hline
\end{tabular}

tive value corresponds to a decrease relative to the normal condition. Because all acoustic measures were computed as difference scores relative to the normal (1OFFPRE) condition, the initial perturbing effects of the palate are reflected in the magnitudes of the bars in the graph. Statistical analyses focused on a comparison of these difference magnitudes across palate conditions (thin, thick) and practice intervals (pre-, post-). Statistical results for the acoustic measures are summarized in Table I.

A series of Palate (thin, thick) $\times$ Practice (pre-, post-) ANOVAs for each consonant and each measure revealed no main effect of Palate, suggesting somewhat surprisingly that both thin and thick palates yielded similar acoustic effects (see Fig. 5). As it is clear from the large SD shown in the Fig. 5, there was a good deal of individual variability, which may account, in part, for the absence of a significant Palate effect. To illustrate, in the production of [s], six subjects surprisingly increased center of gravity in the thick palate condition compared with the thin palate condition with a mean increase of $67 \mathrm{mel}$, while three subjects ${ }^{3}$ decreased center of gravity by $146 \mathrm{mel}$ on average. These acoustic findings suggest different strategies on the part of individual speakers in adaptation to the palatal perturbation (see also Aasland et al., 2006; Baum and McFarland, 2000). Figure 5 also shows that the $15 \mathrm{~min}$ of target-specific practice resulted in substantial changes in [s] acoustic values; center of gravity was lower in both perturbed conditions before practice, while it approached normal values after practice. A significant main effect of practice was found on the center of gravity measures for [s] $[F(1,8)=6.139, p \leq 0.05]$. The interaction of practice and palate conditions was also significant $[F(1,8)=8.117, p \leq 0.05]$. Post hoc analysis revealed that $[\mathrm{s}]$ center of gravity differed significantly in the two thick palate conditions $(p \leq 0.05)$. In fact, it reached zero in the 4THICKPOST condition, indicating that complete adaptation occurred after training with the thick palate along this acoustic dimension. A significant effect of practice also emerged for [s] standard deviation, which was reduced after practice $[F(1,8)=6.092, p \leq 0.05]$; a similar pattern emerged for [ $[$ s] , with SD measures significantly lower after practice $[F(1,8)=24.181, p \leq 0.01]$. For $[\mathrm{t}]$, center of gravity measures were unexpectedly significantly lower after practice $[F(1,8)=6.670, p \leq 0.05]$. The ANOVA revealed no significant acoustic difference for $/ \mathrm{k} /$ since changes are not consistent across subjects.

Figure 6 displays a comparison of 1OFFPRE and 6OFFPOST conditions to assess potential after-effects. A significant 

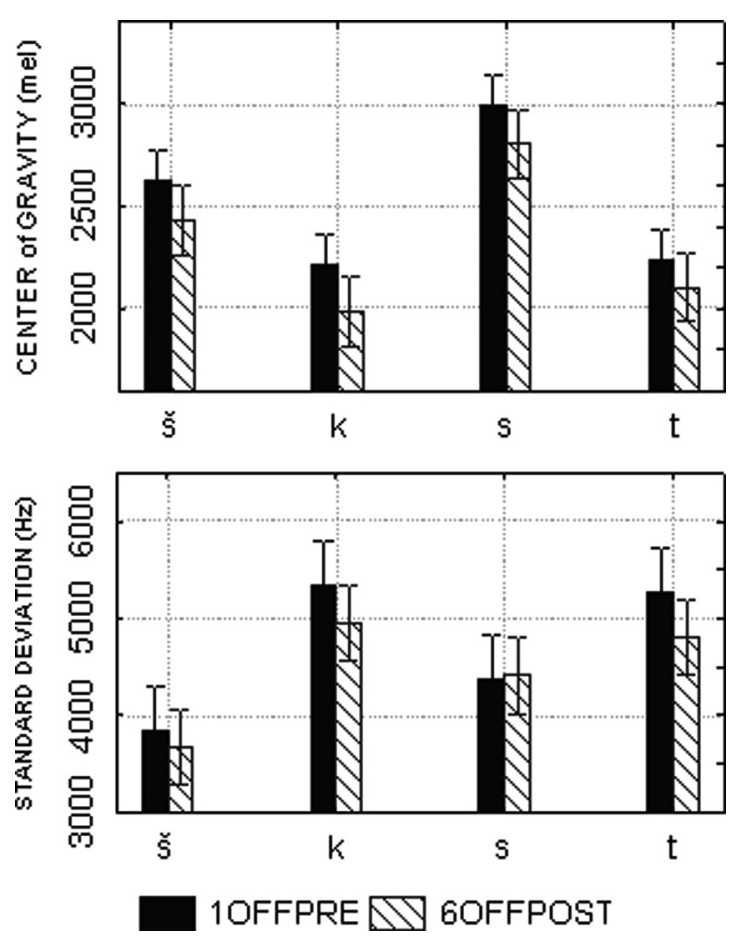

FIG. 6. Average values and SD of center of gravity and SD for the four consonants in the normal condition pre-perturbation (1OFFPRE) and post-perturbation (6OFFPOST).

difference was found only for [s] center of gravity which was lower after the palate was removed $(F(1,9)=21.419, p$ $\leq 0.01$ ), showing there is a rebound effect after the palate removal rather than a typical after-effect, wherein the difference learned would be maintained after perturbation and not in the opposite direction as in this case.

\section{B. Articulatory results}

Table II summarizes the significant effects that emerged from the statistical analyses, and Fig. 7 shows the normalized mean and SD for all articulatory measures.

TABLE II. Results of repeated-measure ANOVAs and paired $t$-test conducted on the articulatory parameters: tongue blade sensor (bladex, bladez), tongue body sensor (bodyx, bodyz), interlabial distance (interlab), jaw sensor (jaw), tongue left lateral sensor (leftlat), tongue tip sensor (tipx, tipz), and protrusion (ulx) $(* p \leq 0.05, * * p \leq 0.01)$.

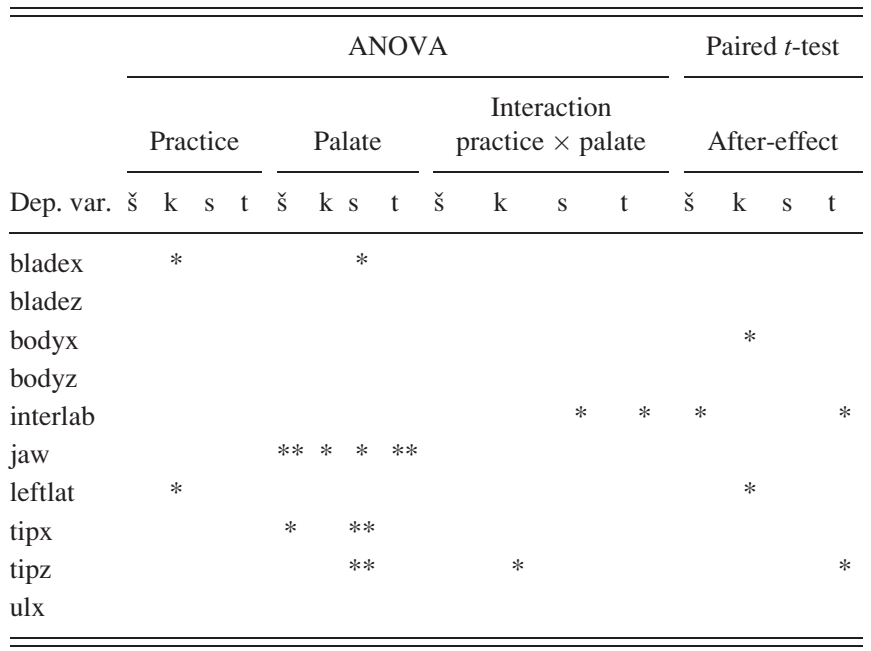

As expected, the most significant effects of the palatal perturbation occurred in [s] production, although all consonants were affected to some degree. For [s], Palate $\times$ Practice ANOVAs yielded significant main effects of Palate for bladex $[F(1,8)=9.170, p \leq 0.05]$, jaw $[F(1,8)=10.892, p$ $\leq 0.05]$, tipx $[F(1,8)=11.316, \quad p \leq 0.01)$, and tipz $[F(1,8)=35.518, p \leq 0.01]$. The jaw was found to be lower with the thick palate as contrasted to the thin palate for $[\mathrm{s}]$ and all other consonants $\{[\check{\mathrm{s}}]: F(1,8)=11.203, p \leq 0.01$; [k]: $F(1,8)=8.563, p \leq 0.05 ;[\mathrm{t}]: F(1,8)=17.206, p \leq 0.01\}$. For [s], bladex and tipx measures were lower and for tipz more back with the thick palate than the thin palate. For [řs], a palate effect was found for tipx $[F(1,8)=8.382, p \leq 0.05]$, the tip being further back with the thick palate than the thin palate. An interaction of Palate $\times$ Practice for the interlabial distance measure $(F(1,9)=7.174, p \leq 0.05)$ was also found for [s]. Post hoc analyses revealed a significant difference only between the 3THICKPRE and 4THICKPOST conditions $(p \leq 0.05)$, with the interlabial distance value smaller after practice. A similar effect is found for the other consonants but it does not reach significance since the effect is less consistent across subjects. Based on a $t$-test between 1OFFPRE and 6OFFPOST conditions, there was no significant after-effect for these parameters.

For [k], there was a significant effect of practice on the bladex measure, which was higher after practice $[F(1,8)=6.320, p \leq 0.05]$. Similarly, a significant effect of practice emerged for the leftLat measure for $[\mathrm{k}]$, reflecting a higher value (more bunched) after practice $[F(1,7)=7.025$, $p \leq 0.05$ ). There was also a significant after-effect in comparisons of 1OFFPRE and 6OFFPOST conditions $[F(1,7)=6.889, \quad p \leq 0.05] \quad$ for this parameter which remained higher after removal of the palate. For [s] production, no significant practice effects emerged for bladex and leftLat due to individual variability across participants; for approximately half of the subjects, the values decreased in the post-practice compared to the pre-practice condition. The remaining minor effects are illustrated in the Table II.

\section{DISCUSSION}

The present study was designed to investigate the effect of palatal perturbation on the production of $[\mathrm{s} \breve{s} \mathrm{t} \mathrm{k}]$ at the articulatory (EMA recordings) and acoustic levels, before and after a 15-min practice interval. One of the key findings was that articulatory movements for [s] production were highly influenced by palatal thickness despite the minimal effects on [s] acoustics. The jaw was lower and the tongue was moved further backwards and downwards for the thick as contrasted to the thin palate. Although it is somewhat surprising that the immediate acoustic effects were of similar magnitude for both the thin and thick palates, the articulatory findings highlight that the two palates did, indeed, induce different compensatory responses. It is likely that the acoustic measures were simply not sensitive enough to capture the differential effects of the thin and thick palates. Alternatively, differences in articulatory movements may not have yielded salient acoustic changes. Both of these potential explanations 

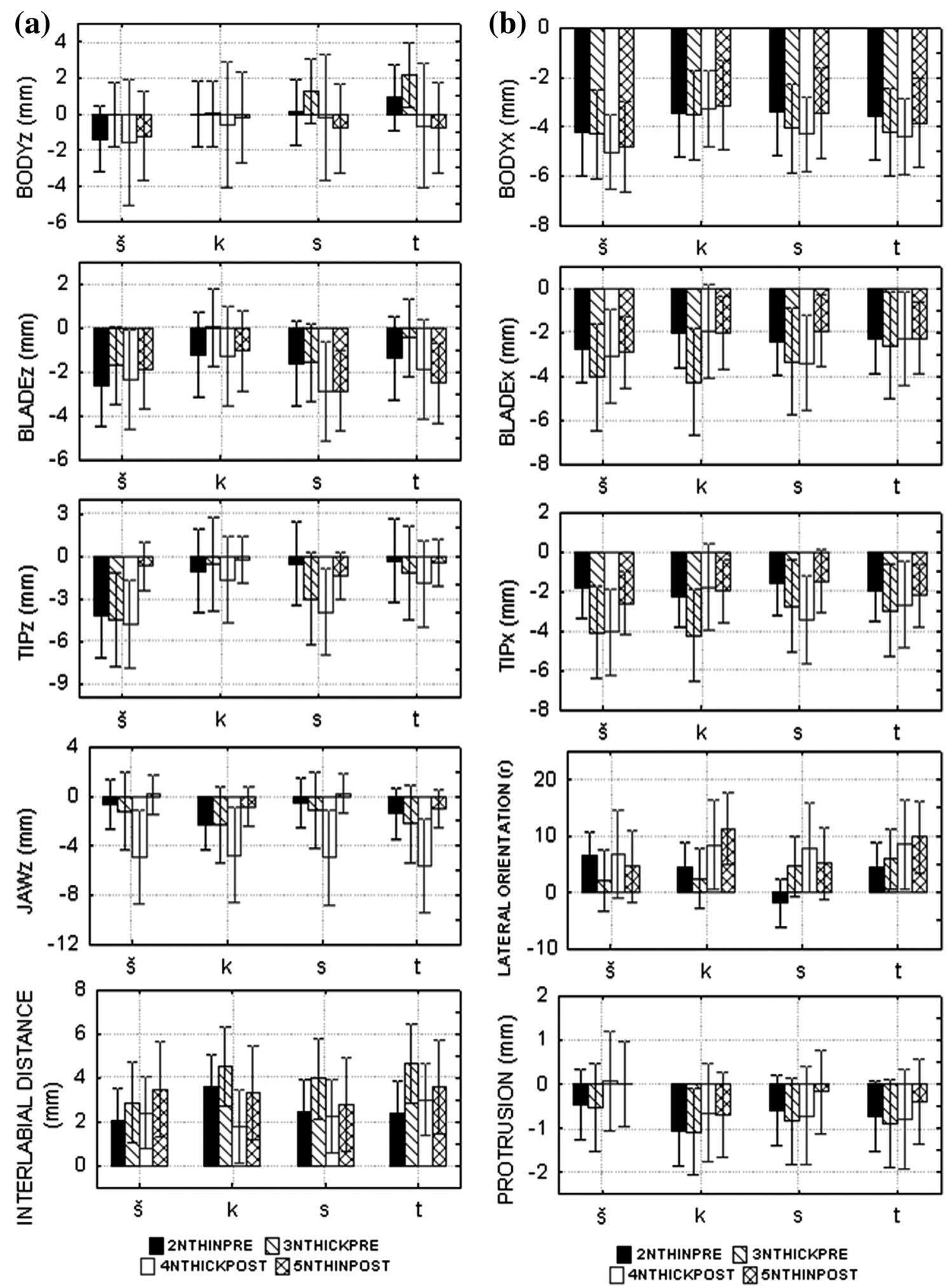

FIG. 7. Average values and SD of within-speaker normalized values of articulatory parameters for the four consonants. Data are shown for the perturbed condition (thin palate pre-perturbation 2NTHINPRE, thick palate pre-perturbation 3NTHICKPRE, thick palate post-perturbation 4NTHICKPOST, thin palate post-perturbation 5NTHINPOST).

argue for the importance of recording both speech acoustics and movement dynamics under conditions of oral-articulatory perturbation - a main focus of the present study.

Another primary goal of the current work was to address issues relevant to speech motor learning. Significant adaptation was observed in [s] acoustics and articulatory measures; for the acoustic measures, [s] center of gravity returned to pre-perturbation values after the practice period with the thick palate in place, suggesting complete adaptation. With respect to articulatory measures, interlabial distance decreased significantly after practice with the thick palate in place for [s], and non-significant decreases were observed in all other 
consonants after training as well. The decrease in interlabial distance opposes the jaw lowering that occurred as an immediate response to insertion of the palate, which appears to be a direct result of the perturbation rather than a compensatory adjustment and is present for all consonants. This may explain the decrease in center of gravity observed in the 3THICKPRE condition. Decreasing the interlabial distance thus appears to represent an adaptive response which serves to normalize (in this case, increase) the acoustic center of gravity. It has been reported that $[\mathrm{s}]$ is normally associated with marginal lateral tongue-palate contact and that this pattern is crucial for the production of the appropriate tongue shape (Flege et al., 1988; Stone et al., 1992). In contrast with [ธ̌], [s] is also associated with a forward constriction location. The airstream is thus filtered by a relatively small resonance cavity formed by the region between the constriction location and the teeth/lips, yielding high spectral frequencies. Although the lateral tongue sensor was higher after practice with the thick palate for [s], indicating that the groove was somewhat smaller, none of the effects for the left lateral sensor orientation reached statistical significance. It is possible that the tongue groove was formed in advance of the tongue making contact with the palate (and stiffened prior to contact) thus explaining the rather small impact on the lateral tongue sensor. The back and low tongue positions observed with the thick palate might have been a strategy to maintain front cavity volume and resonance for the [s] and may have helped the complete compensation for [s] production observed in the center of gravity measures in the acoustic analyses.

Somewhat surprisingly, the only consonant for which articulatory effects of practice reached statistical significance was $[\mathrm{k}]$, which showed a more anterior and perhaps more bunched position after practice. Considering the fact that the palate alone did not significantly affect $/ \mathrm{k} /$ acoustics and the fact that the practice period contained no posterior phonemes, one might interpret this adaptation effect as reflecting a global re-parameterization mechanism in response to perturbation. Additional support for such recalibration comes from the findings of an after-effect for the non-targeted [k] (in tongue lateral orientation), which suggest changes in the underlying motor plans for this speech sound. It is somewhat surprising that the significant adaptation effects were reflected in only a limited number of sensors, perhaps because of between subject variability. Future investigations which permit a more detailed analysis of tongue configuration along its length and width may provide a more sensitive evaluation of the articulatory adjustments in speech motor adaptation to palatal perturbation.

To summarize, the current findings extend those of previous acoustic and perceptual studies by identifying those articulatory movements associated with speech adaptation (in the acoustic domain) to palatal perturbation. The measurement of articulatory movements, as contrasted to tongue-palate contact and/or speech acoustics, provided a more direct assessment of speech movement control in the face of perturbation. Our experimental protocol involved target-specific articulatory practice designed to accelerate speech compensation to oral-articulatory perturbation. Although the impact of the palatal perturbation is complex, implicating both motor output and somatosensory feedback, significant compensatory effects were observed, supporting the claim in the growing body of experimental literature that the speech production system is highly flexible and considerable reorganization can be observed in a brief period of target-specific practice. Clearly, the sensorimotor system is highly interactive, fluidly remapping somatosensory input with the predicted acoustic consequences of motor output to achieve perceptible speech. The present findings also contribute to the growing body of literature suggesting the potential for considerable brain reorganization in the face of targeted and intensive exercise and practice (Kleim and Jones, 2008; Ludlow et al., 2008). The persistence of adaptive effects (i.e., "after-effects") and the impact on sounds whose articulatory gestures are not directly impeded by the presence of the palate suggest a more global re-parameterization of speech movements. Adaptation, therefore, may be tapping into core elements of speech motor scaling that in turn distribute across speech gestures. The present results also point to the potentially important factor of individual differences in compensatory abilities and how sensory feedback interacts with motor performance and structural differences in oral-articulatory structures across experimental subjects. The fundamental and clinical implications of these changes in sensorimotor scaling within and across individuals are significant and deserve additional experimental attention. In a next experiment, it would be interesting to combine auditory perturbation with palatal perturbation to try to better understand how motor and sensory goals impact on speech production, and to more fully characterize individual differences.

\section{ACKNOWLEDGMENTS}

This work was supported by a scholarship from the Social Sciences and Humanities Research Council of Canada (SSHRC) to the first author, a grant from the Natural Sciences and Engineering Research Council of Canada (NSERC) to the second author and grants from the Canada Foundation for Innovation (CFI) and the Social Sciences and Humanities Research Council of Canada (SSHRC) to the third author. We would like to thank Mark Tiede (Haskins laboratory) for his advice regarding the use of the EMA system and for providing the MATLAB procedures for data treatment. Thanks to Serge Drouin, Tatiana Sylvestre, Corinne Toupin, Jérôme Aubin, Amélie Brisebois, and Annie Brasseur for their assistance during the recordings. Thanks to Erin Vensel for serving as a pilot subject.

\footnotetext{
${ }^{1}$ In the event that the tongue blade sensor fell off during the experiment or presented occasional distortion, we used the nearest suitable sensor for labeling (tongue tip for subjects M2 and F5 and tongue right lateral for F6). Results should nevertheless be comparable between subjects, since these three sensors being so close most probably reach a stable position at a comparable time during the consonant production.

${ }^{2}$ Whereas using such a difference measure method may not take into account perceptual differences across different frequency ranges, it nonetheless represented the most straightforward and consistent way to represent the differences across both acoustic and articulatory measures.

${ }^{3}$ For one subject, in one of the conditions, a technical problem occurred during the recording of the audio signal.
} 
Aasland, W., Baum, S. R., and McFarland, D. H. (2006). "Electropalatographic, acoustic, and perceptual data on adaptation to a palatal perturbation,' J. Acoust. Soc. Am. 119, 2372-2381.

Abbs, J. H., and Gracco, V. L. (1984). "Control of complex motor gestures: Orofacial muscle responses to load perturbations of lip during speech," J. Neurophysiol. 51, 705-723.

Baum, S. R., and McFarland, D. H. (1997). "The development of speech adaptation to an artificial palate," J. Acoust. Soc. Am. 102, 2353-2359.

Baum, S. R., and McFarland, D. H. (2000). "Individual differences in speech adaptation to an artificial palate," J. Acoust. Soc. Am. 107, 3572-3575.

Carstens Medizinelektronik (2006). AG500 Manual, 26 p. (http://www. ag500.de/manual/ag500/AG500_manual.pdf).

Chang, S. E., Kenney, M. K., Loucks, T. M., Poletto, C. J., and Ludlow, C. L. (2009). "Common neural substrates support speech and non-speech vocal tract gestures," Neuroimage 47(1), 314-325.

Elman, J. L. (1981). "Effects of frequency-shifted feedback on the pitch of vocal productions," J. Acoust. Soc. Am. 70, 45-50.

Flege, J. E., Fletcher, S. G., and Homiedan, A. (1988). "Compensating for /s/ and / /s/ production: Palatographic, acoustic and perceptual data," J. Acoust. Soc. Am. 83, 212-228.

Gracco, V. L., and Abbs, J. H. (1985). "Dynamic control of the perioral system during speech: Kinematic analyses of autogenic and nonautogenic sensorimotor processes," J. Neurophysiol. 54, 418-432.

Hamlet, S. L., and Stone, M. (1978). "Compensatory alveolar consonant production induced by wearing a dental prosthesis," J. Phonetics 6, 227-248.

Honda, M., Fujino, A., and Kaburagi, T. (2002). "Compensatory responses of articulators to unexpected perturbation of the palate shape," J. Phonetics 30, 281-302.

Houde, J. F., and Jordan, M. I. (1998). "Sensorimotor adaptation in speech production," Science 279, 1213-1216.
Houde, J. F., and Jordan, M. I. (2002). "Sensorimotor adaptation of speech I: Compensation and adaptation," J. Speech Hear. Res. 45, 295-310.

Katz, W. F., Bharadwaj, S. V., and Stettler, M. P. (2006). "Influences of electromagnetic articulatory sensors on speech produced by healthy adults and individuals with aphasia and apraxia," J. Speech Hear. Res. 49, 645-659.

Kawahara, H. (1995). "Hearing voice: Transformed auditory feedback effects on voice pitch control," in Proceedings of the International Joint Conference on Artificial Intelligence (IJCAI'95), Montreal. Workshop on Computational Auditory Scene Analysis, pp. 143-148.

Kleim, J. A., and Jones, T. A. (2008). "Principles of experience-dependent neural plasticity: Implications for rehabilitation after brain damage," J. Speech Lang. Hear. Res. 51(1), 225-239.

Ludlow, C. L., Hoit, J., Kent, R., Ramig, L. O., Shrivastav, R., Strand, E., Yorkston, K., and Sapienza, C. M. (2008). "Translating principles of neural plasticity into research on speech motor control recovery and rehabilitation," J. Speech Lang. Hear. Res. 51(1), 240-258.

McFarland, D. H., and Baum, S. R. (1995). "Incomplete compensation to articulatory perturbation,” J. Acoust. Soc. Am. 97, 1865-1873.

McFarland, D. H., and Tremblay, P. (2006). "Clinical implications of crosssystem interactions," Semin. Speech Lang. 27, 300-309.

Stone, M., Faber, A., Raphael, L., and Shawker, T. (1992). "Cross-sectional tongue shape and lingua-palatal contact patterns in $[\mathrm{s}],[\mathrm{k}]$, and [1]," J. Phonetics 20, 253-270.

Tremblay, S., Shiller, D. M., and Ostry, D. J. (2003). "Somatosensory basis of speech production," Nature 423, 866-869.

Weismer, G., and Bunton, K. (1999). "Influences of pellet markers on speech production behaviour: Acoustical and perceptual measures," J. Acoust. Soc. Am. 105, 2882-2894. 